\section{CAMBIAMENTI PARTONO DA OBIETTIVI CONCRETI}

\author{
La scienza può contribuire al benessere della nazione \\ solo all'interno di un lavoro di squadra \\ Vannevar Bush
}

In questi mesi si parla tanto di "cambiamenti" che sono e saranno la conseguenza della pandemia. Lo stanno facendo giornalisti, economisti, scienziati, filosofi, sociologi e anche noi medici. Sembra un destino ineluttabile, e più o meno tutti sottolineano l'importanza di potere cogliere in questa pandemia una migliore prospettiva di vita e professionale. Un mondo più equo si legge, più rispettoso dell'ambiente e magari dei diritti delle persone più bisognose. Lo abbiamo fatto anche noi su Medico e Bambino e in diverse occasioni, sottolineando che nel nostro prossimo futuro dovremmo dare dimostrazione, come cittadini e operatori sanitari, di ridefinire i nostri valori, rivolti in particolare ai bisogni di un'intera futura generazione $e^{1,2}$.

Nel frattempo quello che sappiamo è che la pandemia ha reso ancora più evidenti le disparità economiche, sociali e in termini di salute ${ }^{3,4}$. Come è noto che la distribuzione dei vaccini anti-Covid è molto diversa tra i Paesi ricchi e quelli poveri. La pandemia ha creato tra le altre cose un alterato regime alimentare, come riportato nella bella Ricerca pubblicata su questo numero (pag. 315). Potremmo dire quasi inevitabilmente, se le basi del sistema sono quelle che abbiamo vissuto e costruito nell'ultimo secolo, dal dopoguerra in poi. Per parlare di cambiamenti individuali (che per le positive ricadute professionali sono indispensabili) quello che si di$\mathrm{ce}$, in modo molto semplice ma anche efficace, è che questi vanno accettati con una motivazione costruttiva. II primo passo, scrive Gaetano Ruvolo (un executive e life coach) sul numero della rivista Forward completamente dedicata ai cambiamenti ${ }^{5}$, è quello di chiederci "cosa possiamo imparare di nuovo da una data situazione", e nel dirlo cita i bambini che i cambiamenti li sperimentano ogni giorno, sempre pronti ad accoglierli, desiderosi di conoscere tutto quello che per loro è una novità. In realtà i cambiamenti fruttuosi per i bambini dipendono dalla positività o meno di quello che vedono e sperimentano e i pediatri e pedagogisti dicono che dipende molto dalle buone traiettorie che si costruiscono sin dai primi giorni di vita. Se ci pensiamo questo vale, anche se in modo meno irreversibile, anche per l'adulto.

II secondo presupposto (che forse è il vero motore del cambiamento) è quello di passare dal "devo" al "voglio". Ė in sintesi la motivazione che ci fa chiedere "cosa voglio veramente"; ma dovremmo avere in mente che la finalità motivante dovrebbe essere in linea con obiettivi se possibile collegiali e nobili, altrimenti il rischio è quello di prospettare il cambiamento solo inseguendo interessi individuali.

Il terzo atteggiamento proposto è quello di chiederci lle domande propositive hanno sempre un impatto favorevole) quale sia la cosa migliore e più utile ed efficace quando $\mathrm{ci}$ troviamo in una situazione inedita, mai sperimentata, ma che richiede delle risposte rapide, efficienti e qualificate. Possiamo pensare che senza questi presupposti individuali sia difficile immaginare un proficuo cambiamento che abbia una buona resa e una necessaria soddisfazione personale.
Ma questi tre principi indubbiamente non bastano, soprattutto quando parliamo di progetti di salute e di cura. II rischio è quello che ognuno intenda la motivazione al cambiamento secondo una visione di parte, magari egoistica o corporativa e senza necessaria malizia, ma solo perché in questi anni ha vissuto la Sanità e il personale ruolo assistenziale solo e sempre "in un determinato modo".

L'impressione è che in momenti epocali (e non solo) i cambiamenti andrebbero governati da una collettività di persone, partendo dal basso, da una dimensione di ascolto, lontani da trappole di governance che si basano spesso e quasi esclusivamente su decisioni di risparmio o di profitto economico (le due facce di una stessa medaglia), come sono state in gran parte in questi anni. Il cambiamento può richiedere questa modalità innovativa di governance ma può anche prevedere di misurarlo, di studiarlo nel metodo applicativo e indirizzarlo là dove pensiamo sia meglio che sia.

Ognuno di noi ha una sua piccola agenda di auspicabili cambiamenti. Di quelli che ritiene più prioritari, di quelli magari ideologici, o irrealizzabili. Ne vorrei riportare alcuni che riguardano inevitabilmente il nostro mondo pediatrico. Si tratta di una scelta, parziale, e come tale "governata" in questo momento in parte anche dalla inevitabile emotività propositiva.

- Vorrei che la parola "minore" sparisse dal vocabolario dei politici, degli amministratori e anche di noi medici. Ė un termine che ha una sua valenza giuridica ma che di fatto esprime "una sottrazione" che, se ci pensiamo, richiama una maggioranza che per definizione ha i diritti a discapito di una minoranza. In questa fase storica una delle sofferenze maggiori è stata quella di sentire parlare dei "minori" dagli organi di stampa (con rare e lodevoli eccezioni) in merito solo alla loro scolarità. Assistiamo alla "terza ondata" di giornali locali che nelle loro copertine, vicino alle edicole, riportano i manifesti sui nuovi focolai scolastici con le riaperture. Come a dire che sia colpa di qualcuno e che qualcuno deve pagare per avere nella stragrandissima maggioranza dei casi una malattia simile a una banale virosi. Dovrebbe sparire perché in questi giorni quel minore non è colpevolmente agli onori della cronaca e della programmazione socio-sanitaria in risposta a una sofferenza che non ha di fatto trovato risposte pronte da parte della comunità. E il cambiamento auspicato dovrebbe immaginare per i nostri bambini e giovani un futuro post-pandemico che non destini solo "finanziamenti" (senza capire bene dove indirizzarli) e che l'occasione di ora è quella di modelli integrati di prevenzione e cura che prevedano una prioritaria attenzione alle famiglie ma anche, in un ambito socio-sanitario, una necessaria riorganizzazione e valorizzazione di funzioni e soprattutto una ridefinizione di alcune competenze professionali: sviluppare reti di connessioni e di servizi di sostegno con le scuole attraverso figure formate di psicologi, infermieri e di servizi sociali integrati con i singoli ambiti distrettuali; riorientare i servizi sui bisogni di salute (mentale) dei giovani significa prendere atto del fatto che oggi sono caratterizzati da un elevato livello di frammentazione nei metodi, nei luoghi, nelle modalità di interazione, il che spesso comporta paradossalmente la mancata richiesta di aiuto ${ }^{6}$. $E$ una scommessa in positivo e non per sottrazione di una "minoranza", che da troppo tempo e colpevolmente è senza voce e ascolto. Si tratta di ribaltare la 
percezione delle cose: non vedere i bambini e gli adolescenti come residuali ma come punto di partenza?

- E a questo proposito vorrei come cambiamento culturale che si smettesse di parlare unicamente della aprioristica "collocazione" funzionale del variegato mondo che si occupa della salute dei bambini e adolescenti: la Neuropsichiatria che dovrebbe lavorare (antistoricamente) con i Servizi di salute mentale (basta leggere i due contributi pubblicati su questo numero, pagg. 321 e 325 , per capire quelle che devono essere le future prospettive del dialogo e della formazione congiunta tra Pediatria e Neuropsichiatria); la Pediatria di famiglia con la Medicina territoriale; le Pediatrie ospedaliere esclusivamente con i Servizi di Ostetricia e Ginecologia. Una Sanità senza confini ${ }^{8}$ può vivere solo realizzando modelli di integrazione per obiettivi stringenti di miglioramento della salute individuale e collettiva e non per un'appartenenza fatta di rappresentanza e come tale unicamente corporativa o basata su convenienze amministrative o politiche. - Come cambiamento auspicherei che i famosi "Percorsi di budget" che riguardano quasi esclusivamente il mondo ospedaliero (e non si capisce perché!) si chiamassero annualmente "Percorsi di integrazione e di risposta ai bisogni"; e che magari oltre a quello sul "Percorso nascita", ripetitivo, stantio e quasi inutile se si ferma "all'evento nascita" (concetti triti e ritriti), si parlasse di un "Percorso di accompagnamento delle famiglie e dei neonati" con evidenti bisogni di deprivazione sociale; alla cronicità, ma con verifica puntuale di ruoli, funzioni, risposta anche di prossimità ai bisogni e di soddisfazione familiare. Vorrei che si parlasse di "Percorsi per la prevenzione e la definizione delle traiettorie più funzionali" per la migliore crescita (fisica e mentale) dei nostri neonati-lattanti sino a quando non diventeranno adolescenti.

- Vorrei infine che la distinzione tra cosa va cambiato e cosa conservato (in una scelta inevitabilmente selettiva) si basasse su logiche che non prevedano mai il comodo disfattismo nichilista che ci esula dal darci da fare, immaginando che sia sempre colpa degli altri o che tutto cambi tranne i propri privilegi. Solo una idea di Pediatria unita e motivata nel raggiungere obiettivi di cambiamenti concreti può essere la risposta al disfattismo e al conservatorismo.

È chiaro che questi piccoli e personali bisogni di cambiamento sono parziali e in questo momento è difficile dire cosa sia giusto o sbagliato. Ma di sicuro come prospettiva non devono avere barriere e un'appartenenza esclusiva in "luoghi fisici salute" che, se non prevedono ruoli di alto profilo professionale, rischiano di essere contenitori vuoti. Questi cambiamenti dovrebbero basarsi appunto sulla professionalità, sulla rete, sulla comunità funzionale, sulla scommessa forte e imprescindibile basata anche sulle figure infermieristiche, sugli educatori e, quando necessario, su Servizi sociali all'altezza di questo nome.

$\mathrm{Mi}$ è capitato di leggere recentemente un libro ${ }^{9}$ di quelli che ti cambiano in parte la prospettiva di visione nel tuo ruolo di pediatra. A parlare è una madre con due figli colpiti da malattie molto gravi (una con la sindrome di Rett, I'altro con un tumore cerebrale). La sofferenza genitoriale (estrema) si trasforma in energia, progettualità, combustibile da bruciare, "perché quando si ammala un bambino tutti si ammalano, tranne il bambino. Lui non sa di essere malato". Il dolore, anche quello della pandemia, dovrebbe essere un motore catartico per una felicità contagiosa che è una scelta, una scelta di cambiamento, che è in grado di superare in modo consapevole gli ostacoli. L'importante tuttavia è immaginarsi dentro semplici obiettivi concreti, da sperimentare giorno dopo giorno.

\section{Bibliografia}

1. Marchetti F. Una volta che ci saremo ripresi dalla pandemia ridefiniremo i nostri valori? Medico e Bambino 2021;40(2): 109. 11. doi: 10.53126/MEB40109.

2. Ventura A. L'ottavo giorno. Medico e Bambino 2021;40(2):79. doi: $10.53126 /$ MEB 40079.

3. Burström $B$, Tao W. Social determinants of health and inequalities in Covid-19. Eur J Public Health 2020;30(4):617-8. doi: 10.1093/eurpub/ckaa095.

4. Tamburlini $G$. La legge delle cure inverse: un anniversario da ricordare. Medico e Bambino 2021;40(3):145. doi: 10.53126/ MEB40145.

5. AA.VV. Cambiamenti. Foward 21. Supplemento Recent Prog Med 2021;1 12,(4). https://issuu.com/pensiero/docs/21-fwdcambiamenti.

6. Marchetti F. I Servizi di salute mentale per i bambini in Inghilterra (e in Italia) "non sono neanche lontanamente sufficienti". Medico e Bambino 2021;:40(3): 157-8. doi: 10.53126/MEB40157.

7. Tonon L (a cura di). Una nuova idea di fare politica. Ripartire insieme dal territorio con i bambini. Intervista all'onorevole Paolo Siani. Foward 21. Supplemento Recent Prog Med 2021;1 12 (4):223. https://issuu.com/pensiero/docs/2 1-fwd-cambiamenti.

8. Centro per la Salute del Bambino, ACP. Senza confini. Come ridisegnare le cure all'infanzia e all'adolescenza, integrando i servizi, promuovendo l'equita, diffondendo le eccellenze. Medico e Bambino 2020, 20 settembre. https://bit.ly/3gJuGVd [ultimo accesso 29 aprile 2021].

9. Tarì M. II precipizio dell'amore. Solo appunti di una madre. Mondadori editore. Marzo 2021.

Federico Marchetti

\section{BAMBINI NEL PIANO NAZIONALE RIPRESA E RESILIENZA}

Come solennemente ribadito sia dalla Commissione Europea (CE) che dal presidente del Consiglio, i fondi del Next Generation EU (NGEU) "hanno la finalità di costruire un'Europa e un'Italia migliore soprattutto per le nuove generazioni". II Piano Nazionale di Ripresa e Resilienza (PNRR) predisposto "dopo un lungo e contrastato lavoro a cavallo di due successivi Governi" è stato alla fine approvato dalle Camere e inviato alla CE. Logico chiedersi se risponde adeguatamente alle finalità dichiarate. Si tratta di un documento complesso (336 pagine), dove sarebbe errato andare a cercare le implicazioni per le nuove generazioni solo dove queste ultime vengono esplicitamente citate. È chiaro infatti che un ambiente più sano, una pubblica amministrazione più efficiente, una società più inclusiva e meno diseguale, e migliori prospettive occupazionali sono nell'interesse di tutti ma soprattutto di coloro che, avendo una prospettiva di vita più lunga, dovranno portare il peso di un pianeta più malato $e$ in Italia, di un debito pubblico crescente. Tuttavia sarebbe pure illusorio pensare che il PNRR possa essere di beneficio per i giovani se i capisaldi delle politiche a favore di infanzia e famiglie - educazione, salute 
e protezione sociale - fossero trascurati. A questi tre settori sono dedicate tre delle sei "missioni" in cui è strutturato il PNRR (digitalizzazione e innovazione, transizione ecologica, infrastrutture per la mobilità sostenibile, istruzione e ricerca, inclusione e coesione sociale, salute). Innanzitutto, risorse consistenti (19,4 miliardi) sono destinate all'istruzione, di cui 4,6 per "aumentare significativamente (il testo indica che tale misura consentirà la creazione di circa 228.000 nuovi posti) l'offerta negli asili nido e nelle scuole dell'infanzia". Le rimanenti risorse vanno al resto del percorso scolastico dalla primaria all'università, per investimenti sul personale e la sua formazione (degna di nota la previsione di una scuola superiore per i dirigenti scolastici), sulle infrastrutture e le dotazioni tecnologiche, con l'obiettivo generale di incrementare le competenze degli studenti e minimizzare dispersione e abbandono. Viene ribadito, e accompagnato da risorse (1 1,7 miliardi), il sostegno alle politiche e ai servizi sociali e si fa esplicito riferimento al potenziamento dei programmi per le famiglie vulnerabili e al ruolo dei servizi sociali come strumento di resilienza, "mirando alla definizione di modelli personalizzati per la cura delle famiglie, dei minori e degli adolescenti" (in merito, non va dimenticato che il Parlamento ha finalmente approvato in aprile l'assegno unico per i figli). Dei 15,6 miliardi investiti in salute, 7 vanno alle strutture territoriali e gli altri a innovazione ricerca e digitalizzazione. Per il territorio, si prevede la creazione di 1226 "Case di comunità" dove devono trovare posto (funzionale e/o logistico) tutti i servizi territoriali e quindi anche quelli per donne e bambini.

Infanzia, adolescenza e giovani quindi "ci sono", nel PNRR, e la loro centralità è specificamente sottolineata. Tutto questo rappresenta un fatto nuovo e importante. Tuttavia, le richieste giunte in questi ultimi mesi dalla società civile e dallo stesso Parlamento ${ }^{1-4}$ erano superiori, e più precise. Se è comprensibile che un Piano da oltre 200 miliardi che comprende anche indicazioni per riforme strutturali (amministrazione, giustizia, fisco) non entri nel dettaglio, alcuni rilievi sono necessari: 1) 226.000 nuovi posti in nido in 5 anni non sono assolutamente sufficienti a raggiungere nemmeno l'obiettivo minimo del $33 \%$ di copertura su tutto il territorio nazionale, cioè in ciascuna Regione e non in media; 2) la necessità del "sostegno alle competenze e risorse genitoriali" viene sottolineata ma non tradotta in indicazioni sui servizi da attivare; 3) per i bandi pubblici finalizzati alla creazione di nuovi servizi per l'infanzia e le famiglie, non sono indicati parametri per favorire le zone più svantaggiate né un meccanismo di supporto alla progettazione e gestione dei servizi, in mancanza del quale si finisce con privilegiare le realtà dove sono già presenti sia tali competenze che la capacità di sostenere la componente corrente della spesa; 4) l'investimento sulle Case della salute (denominazione preferibile!) dice ancora poco di quello che conta: con quale personale, con quale gestione e quale ruolo per la Medicina e la Pediatria di famiglia, la cui attuale organizzazione, ancora prevalentemente basata sull'ambulatorio individuale e troppo poco integrata con i restanti servizi, è del tutto superata; 5) per le problematiche relative all'adolescenza - e in particolare per la salute mentale, che non riguarda solo l'adolescenza, ma che in que- sta età chiave ha un suo punto di particolare criticità - il PNRR spende poche parole e non prevede investimenti specifici. Infine, come richiesto da tutti coloro che si occupano di infanzia, occorre procedere a definire un Piano nazionale per l'infanzia e l'adolescenza che integri gli interventi dei diversi settori a livello nazionale come locale ${ }^{1,2 \cdot 4}$. In generale, e non solo per quanto riguarda i bambini, se non si può pretendere che un piano della portata e complessità del PNRR (e da approvarsi in tempi rapidi) preveda anche dettagli attuativi, certamente occorre definire meccanismi per cui se alcuni Enti sono inadempienti nella realizzazione di quanto previsto, subentra una gestione esterna. La pandemia ha infatti insegnato che si sono tollerati troppo i potentati e le incompetenze locali: non è accettabile che le popolazioni paghino l'incapacità di spendere bene. II PNRR dice che questo verrà fatto, e che è questa l'agenda dei prossimi due-tre anni, a partire dal rafforzamento e rinnovamento della pubblica amministrazione, a tutti i livelli.

In sintesi: il PNRR si presta (a una lettura benevola) a essere visto come interprete di una impostazione del tipo Children in all policies ${ }^{5}$, dove cioè si riconosce che i bambini staranno meglio (non solo di salute) se un contributo verrà da tutti i settori; e riconosce, e affronta, alcune criticità (non tutte) e prevede investimenti importanti (ma ancora non sufficienti). Molto viene affidato agli Enti attuatori, centrali e locali, con le disparità e ritardi che ne possono seguire.

La misura del successo complessivo del PNRR sarà senza dubbio data soprattutto dai classici indicatori economici (PIL, debito pubblico, distribuzione del reddito, occupazione ecc.). Ma, per quanto riguarda i bambini, gli indicatori ultimi di una sua efficace attuazione, saranno la fine, se non l'inversione, del trend negativo della natalità e la diminuzione dell'emigrazione di giovani, soprattutto qualificati. Se il Paese vedrà una prospettiva, la vedranno soprattutto i giovani. E se loro staranno meglio, non c'è dubbio che nascerà qualche bambino in più. Vedremo...

\section{Bibliografia}

1. Rete CRC. I diritti dell'infanzia e dell'adolescenza in Italia. $11^{\circ}$ Rapporto di aggiornamento sul monitoraggio della Convenzione sui diritti dell'intanzia e dell'adolescenza in Italia. 20 novembre 2020.

2. Centro per la Salute del Bambino e Associazione Culturale Pediatri. Senza confini: come ridisegnare le cure per l'infanzia e l'adolescenza, integrando i servizi, promuovendo l'equità, diffondendo le eccellenze, 2020.

3. Alleanza per I'Infanzia e EducAzioni. Investire nell'infanzia: prendersi cura del futuro a partire dal presente, 2020. https:// www alleanzainfanzia. it/pubblicato-il-rapporto-investirenellinfanzia-di-alleanza-e-educazioni/

4. Camera dei Deputati. 1-00405 - Mozione sul mancato supporto a bambini ed adolescenti, del Governo nel gestire la pandemia. Aprile 2021.

5. Dalglish SL, Costello A, Clark H, Coll-Seck A. Children in All Policies 2030: a new initiative to implement the recommendations of the WHO-UNICEF-Lancet Commission. Lancet 202 1;397(10285): 1605-7. doi: 10.1016/S0140-6736(21)00718-2.

Giorgio Tamburlini 